Case report

\title{
Atypical presentation and prolongation of retroperitoneal abscess after emergency surgery for perforated ascending colon cancer in a patient receiving anti-interleukin-6 receptor monoclonal antibody therapy
}

\author{
Toshichika Kanagawa $^{1,2)}$, Hiromichi Maeda ${ }^{1)}$, Ken Okamoto $^{3)}$, Ian Fukudome ${ }^{1)}$, Sachi Tsuda ${ }^{1)}$, \\ Tsutomu Namikawa $^{1)}$, Michiya Kobayashi ${ }^{3)}$, Kazuhiro Hanazaki ${ }^{1)}$ \\ ${ }^{1)}$ Department of Surgery, Kochi Medical School, Kochi University \\ ${ }^{2)}$ Department of Surgery, Shikoku Central Hospital \\ ${ }^{3)}$ Cancer Treatment Center, Kochi Medical School Hospital
}

\begin{abstract}
Tocilizumab is an anti-interleukin-6 receptor monoclonal antibody which was recently approved for the treatment of inflammatory arthritis and Castleman disease. Because tocilizumab suppresses immunological reactions, the more frequent occurrence of infections with atypical presentations is a conceivable complication after abdominal surgery. Herein, we would like to describe a case of a prolonged abdominal abscess with subtle abdominal pain after surgery for perforated ascending colon cancer in a patient with Castleman disease treated by tocilizumab.
\end{abstract}

Keywords: Tocilizumab, Castleman, abscess, surgery

(Received August 5, 2019; Accepted August 30, 2019)

\section{Introduction}

Castleman disease is a rare condition with non-neoplastic lymphadenopathy, for which glucocorticoid and cyclosporine are often used $^{1,2)}$. In addition to this conventional treatment, monoclonal antibodies targeted at the interleukin (IL)-6 receptor (tocilizumab) show clinical efficacy ${ }^{3}$. However, tocilizumab is a newly-approved drug and experience of its use during the perioperative period is limited. Herein, we would like to describe a patient with idiopathic Castleman disease treated by tocilizumab. The patient underwent emergency surgery for perforated colorectal cancer and developed a retroperitoneal abscess with significantly delayed healing. We consider that the characteristic features and clinical course of this patient are educational and worthwhile reporting.

\section{Ethical Statement}

The written informed consent was obtained from the patient for the publication of the case.

Address for correspondence: Hiromichi Maeda, MD, PhD. Department 0 Surgery, Kochi Medical School, Kochi University. Kohasu, Oko-cho, Nankoku-city, Kochi, 783-8505, Japan. E-mail; hmaeda@kochi-u.ac.jp, Tel: +81-88-880-2182, Fax: +81-88-880-2183

\section{Case presentation}

A 60-year-old man with a medical history of Castleman disease over 20 years was referred to our department for the treatment of ascending colon cancer. The patient was on tocilizumab $(8 \mathrm{mg} / \mathrm{kg}$ intravenous infusion, every four weeks) and oral steroid treatment (prednisolone, $2 \mathrm{mg}$, everyday). He started to complain of post-prandial abdominal pain a few months beforehand, and a colonoscopy revealed a circumferential cancer in his ascending colon. Due to stenosis, he was admitted to our department for fasting and intravenous infusion therapy prior to elective surgery. However, he developed acute-onset abdominal pain with a diagnosis of colonic perforation (Fig. 1A), requiring emergency right hemicolectomy (Fig. 1B).

Initially, the patient's recovery seemed uneventful (Fig. 2). The abdominal drainage tubes placed at Douglas's pouch and right subphrenic space were removed three days after surgery and the tubes at left subphrenic space and retroperitoneal space (dorsal side of the anastomosis) were removed five days after operation because only small amount of the serous drainage fluid was observed. Twelve days after the operation, the patient started to complain of a mild right abdominal pain. Computed tomography revealed retention of fluid in the right retro- 

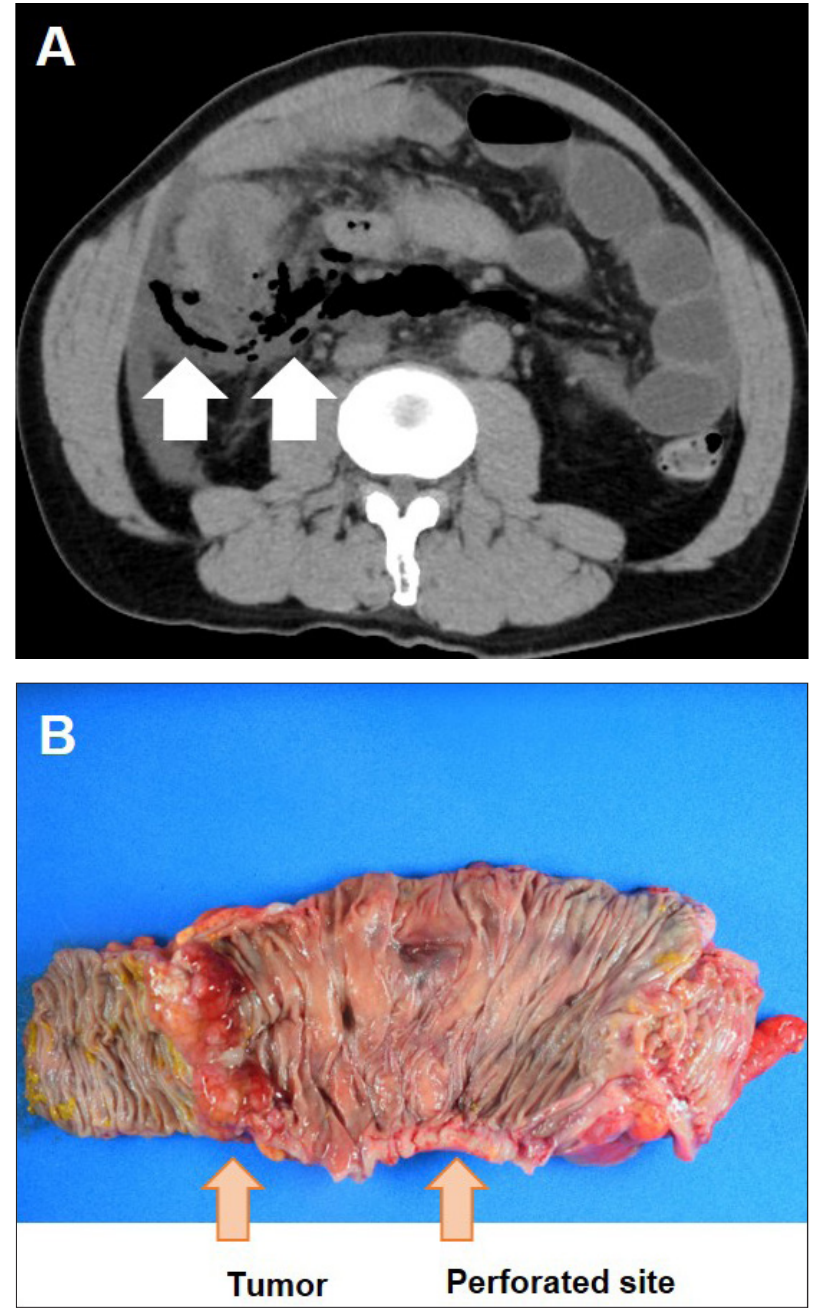

Fig. 1 (A) Computed tomography revealed free air on the dorsal side of the superior mesenteric artery (retroperitoneal space, white arrow). (B) Resected specimen of the colon, showing the circumferential colon cancer at the ascending colon. The perforation was not in the cancer itself but located at the oral side of the cancer.
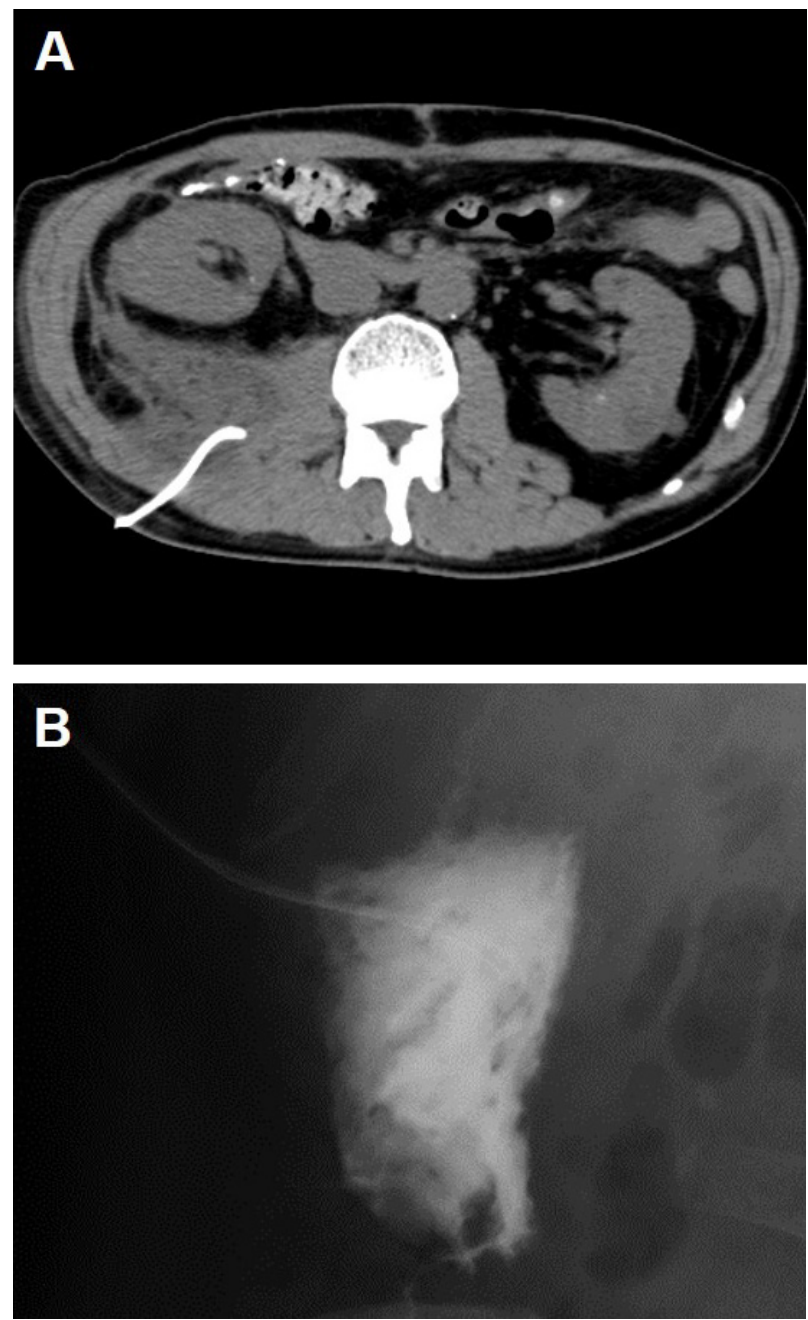

Fig. 3 (A) A drainage tube was placed. (B) After radiological examination, which revealed no connection between the cavity and anastomosis, the abscess was continuously washed with normal saline.

Fig. 2 The clinical course of the present case is shown with the transition of C-reactive protein (CRP) in the serum, and with the presentation of computed tomography (CT) scans. Thirteen days before emergency surgery, the patient received tocilizumab treatment. After the operation (Day 0), CRP markedly increased to $13 \mathrm{mg} /$ $\mathrm{dL}$, then decreased within a few days. When the patient complained of subtle abdominal pain, CRP was only 0.87 $\mathrm{mg} / \mathrm{dL}$. Abdominal CT revealed a collection of fluid on the dorsal side of his right kidney (upper panel). Although his CRP was slightly higher than the normal range, a marked increase in CRP was not observed afterwards. At the second $\mathrm{CT}$, the distended fluid collection with enhancement of the wall suggests the formation of a retroperitoneal abscess (lower panel), despite the decreased level of serum CRP. 
peritoneal space (around the right kidney) without free air. His serum C-reactive protein (CRP) remained low at $1.09 \mathrm{mg} / \mathrm{dL}$, and the pain gradually eased without treatment. Furthermore, no increase in the fluid on abdominal ultrasonography was seen, and the patient was discharged after receiving a dose of tocilizumab (Fig. 2).

One week after discharge, the patient remained afebrile and the severity of his abdominal pain was similar to that at discharge, and his level of serum CRP had decreased to $0.16 \mathrm{mg} / \mathrm{dL}$. However, abdominal ultrasonography and computed tomography showed the retention of fluid had increased and enhancement of the surrounding wall was observed, suggesting the development of a retroperitoneal abscess. The patient was re-admitted for echo-guided drainage with placement of a drainage tube (Fig. 3A), which removed whitish pus. The pain quickly subsided, and a follow-up radiological examination revealed no anastomotic leakage (Fig. 3B). Although the abscess was continuously flushed with normal saline, the abscess cavity remained and it was 41 days before the drainage tube was removed. After the second discharge, there has been no signs of recurrence of the abscess or colon cancer.

\section{Discussion}

Castleman disease is a rare condition with nonneoplastic lymphadenopathy, in which increased release of IL- 6 is related to the activity of the disease ${ }^{1,2)}$. Most patients with idiopathic Castleman disease require treatment for the accompanying symptoms, such as weight loss, fatigue, night sweats and malaise ${ }^{4)}$. Glucocorticoid and cyclosporine are treatment options for idiopathic Castleman disease; however, in patients with disease resistance against these conventional treatments, monoclonal antibodies targeted at IL- 6 or the IL- 6 receptor (tocilizumab) show clinical efficacy resulting in alleviation of symptoms ${ }^{1-3)}$.

Tocilizumab is a relatively newly-approved drug and reports on its use during the perioperative period are scarce. The present case demonstrates two important clinical features of abdominal abscesses in patients receiving tocilizumab treatment. First, the patient complained only of subtle abdominal pain, which was not accompanied by an increase in CRP or fever. IL- 6 is an inflammatory cytokine and important for the host defense in the case of infection or surgical stress. Local pain is partially controlled by IL-6, as well as tumor necrosis factor- $\alpha$ and IL- $1^{5)}$. CRP is produced mainly in the liver via the signaling of IL- ${ }^{6}$ ) and participates in the systemic response to inflammation. In the present case, the use of tocilizumab suppressed these IL- 6 functions and lessened the systemic response (pain, fever, and serum level of CRP), even after the development of the retroperitoneal abscess. Thus, we strongly recommend closely monitoring patients after abdominal surgery and invasive diagnostic methods should be considered if needed. Furthermore, any possible lesion should be carefully monitored until disappearance of the lesion.

The second important clinical feature of this present case was the delayed healing of the abdominal abscess. In addition to its role in host defense, IL- 6 is known to have a crucial role in bone regeneration and corneal wound healing ${ }^{7,8)}$. Furthermore, delayed wound healing after surgical interventions, such as foot and spinal surgery, has been reported in patients treated with tocilizumab $^{9)}$. Therefore, we consider that the removal of the drainage tube should be delayed, and the possibility of recurrence of the abscess should be explained to the patient for earlier detection of any recurrence.

In the present case, the patient received $2 \mathrm{mg}$ of oral prednisolone, which has immunosuppressive effects and may have concealed the symptoms related to the abscess formation. Because tocilizumab is generally used in patients whose disease is resistant against conventional therapy, the combination therapy of the tocilizumab and steroid is not uncommon practice. We consider the more cautious postoperative monitoring of the symptom and laboratory tests are required in such cases.

In conclusion, close monitoring after abdominal surgery is required in patients treated with tocilizumab, because these patients may not present with the classical symptoms of postoperative complications. Also, complete eradication of infectious lesions is required because delayed healing may occur.

\section{Conflict of interest:}

There is no conflict of interest to declare.

Financial support: None

\section{References}

1) Koga T, Sumiyoshi R, Kawakami A, Yoshizaki K. A benefit and the prospects of IL-6 inhibitors in idiopathic multicentric Castleman's disease. Mod Rheumatol. 2019; 29:302-305.

2) van Rhee F, Greenway A, Stone K. Treatment of Idiopathic Castleman Disease. Hematol Oncol Clin N Am. 2018; 32:89-106.

3) Song SN, Tomosugi N, Kawabata H, Ishikawa T, Nishikawa T, Yoshizaki K. Down-regulation of hepcidin resulting from longterm treatment with an anti-IL-6 receptor antibody (tocilizumab) improves anemia of inflammation in multicentric Castleman disease. Blood. 2010; 116:3627-3634.

4) Haap M, Wiefels J, Horger M, Hoyer A, Müssig K. Clinical, laboratory and imaging findings in Castleman's disease - The subtype decides. Blood Rev. 2018; 32:225-234.

5) Rose-John S, Scheller J, Elson G, Jones SA. Interleukin-6 biology is coordinated by membrane-bound and soluble receptors: role in inflammation and cancer. J Leukoc Biol. 2006; 80:227-236.

6) Black S, Kushner I, Samols D. C-reactive protein. J Biol Chem. 2004; 279:48487-48490.

7) Prystaz K, Kaiser K, Kovtun A, Haffner-Luntzer M, Fischer V, Rapp AE, et al. Distinct effects of IL-6 classic and trans-signaling in bone fracture healing. Am J Pathol. 2018; 188:474-490. 
8) Ebihara N, Matsuda A, Nakamura S, Matsuda H, Murakami A Role of the IL-6 classic- and trans-signaling pathways in corneal sterile inflammation and wound healing. Invest Ophthalmol Vis Sci. 2011; 52:8549-8557.
9) Momohara S, Hashimoto J, Tsuboi H, Miyahara H, Nakagawa N, Kaneko A, et al. Analysis of perioperative clinical features and complications after orthopaedic surgery in rheumatoid arthritis patients treated with tocilizumab in a real-world setting: results from the multicentre TOcilizumab in Perioperative Period (TOPP) study. Mod Rheumatol. 2013; 23:440-449. 